H. Morikawa

Nagoya Math. J.

Vol. 48 (1972), 183-188

\title{
A NOTE ON HOLOMORPHIC VECTOR BUNDLES OVER QUOTIENT MANIFOLDS WITH RESPECT TO NILPOTENT GROUPS
}

\author{
HISASI MORIKAWA
}

1. A holomorphic vector bundle $E$ over a complex analytic manifold $\mathscr{D}$ is said to be simple, if its global endomorphism ring $\operatorname{End}_{C}(E)$ is isomorphic to $C$. Projectifying the fibers of $E$, we get the associated projective bundle $P(E)$ of $E$. If we can choose a system of constant transition functions of $P(E)$, the projective bundle $P(E)$ is said to be locally flat.

In the present note we shall prove the following the theorem:

THEOREM 1. Let $\Gamma$ be a finitely generated nilpotent subgroup in the group of automorphisms of a complex analytic manifold $\mathscr{D}$. Assume that $\Gamma$ acts properly discontinuously on $\mathscr{D}$ without fixed points. Let $E$ be a holomorphic vector bundle over the quotient manifold $\mathscr{D} / \Gamma$ such that i) the inverse image of $E$ with respect to the natural map $\mathscr{D} \rightarrow \mathscr{D} / \Gamma$ is trivial, ii) the associated projective bundle $P(E)$ is locally flat and iii) $E$ is simple. Then there exists a subgroup $\Delta$ of finite index in $\Gamma$ and a line bundle $L$ over the quotient $\mathscr{D} / \Delta$ such that $E$ is isomorphic to the direct image of $L$ with respect to the natural map $\mathscr{D} / \Delta \rightarrow \mathscr{D} / \Gamma$.

A complex nilmanifold is defined as the quotient of simply connected nilpotent complex Lie group $G$ with respect to a discrete subgroup $\Gamma$ of $G$. The finiteness of $\operatorname{dim} G$ implies the finite generation of $\Gamma$, and $G$ is biholomorphic to a complex vector space. Hence, applying Theorem 1 to $\mathscr{D}=G$, we conclude that

THEOREM 2. Let $\Gamma$ be a discrete subgroup in a simply connected nilpotent complex Lie group $G$. Let $E$ be a holomorphic vector bundle

Received January 19, 1972.

Revised May 30, 1972. 
over the nilmanifold $G / \Gamma$ such that i) the associated projective bundle $P(E)$ is locally flat and ii) $E$ is simple. Then there exists a subgroup $\Delta$ of finite index in $\Gamma$ and a line bundle $L$ over $G / \Delta$ such that $E$ is isomorphic to the direct image of $L$ with respect to the natural map G/S $\rightarrow G / \Gamma$.

2. We need two algebraic lemmas.

LEMMA 1. Let $\Gamma$ be a finitely generated nilpotent group and let $Z$ be its center. If the exponent of $Z$ is finite, then $\Gamma$ is a finite group.

Proof. First we show that the exponent of $\Gamma$ is finite. Denote by

$$
Z^{(r)}=\Gamma \supset Z^{(r-1)} \supset \cdots \supset Z^{(1)} \supset Z^{(0)}=\{1\}
$$

the upper central series of $\Gamma$. By the assumption the exponent of $Z^{(1)} / Z^{(0)}$ is finite. Assume that the exponent of $Z^{(s)} / Z^{(s-1)}$ is finite, say $n$. Since $\left(\Gamma, Z^{(s+1)}\right) \subset Z^{(s)}$ and $\left(\Gamma, Z^{(s)}\right) \subset Z^{(s-1)}$, it follows that for $a \in Z^{(s+1)}$ and $b \in \Gamma$

$$
\begin{array}{cc}
a^{-1} b^{-1} a=(a, b) b^{-1}, & (a, b) \in Z^{(s)}, \\
a^{-1}(a, b) a \equiv(a, b) & \bmod Z^{(s-1)} .
\end{array}
$$

Hence

$$
a^{-n} b^{-1} a^{n} \equiv(a, b)^{n} b^{-1} \equiv b^{-1} \quad \bmod Z^{(s-1)}
$$

and thus

$$
a^{n} b \equiv b a^{n} \quad \bmod Z^{(s-1)} .
$$

This means that $a^{n} \in Z^{(s)}$ for $a \in Z^{(s+1)}$ and the exponent of $Z^{(s+1)} / Z^{(s)}$ is finite. Therefore the exponents of $Z^{(s)} / Z^{(s-1)}(1 \leq s \leq r)$ are finite and consequently the exponent of $\Gamma$ is finite. To prove the finiteness of the order of $\Gamma$, we need the lower central series

$$
\Gamma=\Gamma_{(0)} \supset \Gamma_{(1)} \supset \cdots \supset \Gamma_{n}=\{1\} .
$$

Since $\Gamma / \Gamma_{(1)}$ is a finitely generated abelian group and its exponent is finite, the group $\Gamma / \Gamma_{(1)}$ is a finite group. Assume that $\Gamma / \Gamma_{(s)}$ is a finite group. It is enough to show that $\Gamma / \Gamma_{(s+1)}$ is also a finite group. Let $\left\{\bar{a}_{1}, \cdots, \bar{a}_{m}\right\}=\Gamma / \Gamma_{(s)}$ and $\left\{\bar{b}_{1}, \cdots, \bar{b}_{l}\right\}=\Gamma_{(s-1} / \Gamma_{(s)}$. Let $\left\{a_{1}, \cdots, a_{m}\right\}$ and $\left\{b_{1}, \cdots, b_{l}\right\}$ be representatives of $\left\{\bar{a}_{1}, \cdots, \bar{a}_{m}\right\}$ and $\left\{\bar{b}_{1}, \cdots, \bar{b}_{l}\right\}$ in $\Gamma / \Gamma_{(s+1)}$. Since $\Gamma_{(s)} / \Gamma_{(s+1)}$ is contained in the center of $\Gamma / \Gamma_{(s+1)}$, the commutators $\left(a_{i}, b_{j}\right)(1 \leq i \leq m, 1 \leq j \leq l)$ do not depends on the choice of the repre- 
sentatives. This shows that $\Gamma_{(s)} / \Gamma_{(s+1)}$ is an abelian group generated by $\left(a_{i}, b_{j}\right)(1 \leq i \leq m, 1 \leq j \leq l)$ and its exponent is finite. Hence $\Gamma_{(s)} / \Gamma_{(s+1)}$ is a finite group, and thus $\Gamma / \Gamma_{(s+1)}$ is a finite group. This completes the proof of Lemma 1 .

LEMMA 2. Let $\tilde{\Gamma}$ be a nilpotent subgroup in $G L(n, C)$ and let $\tilde{Z}$ be its center. Assume that $\tilde{\Gamma} / \tilde{Z}$ is finitely generated and the commutor of $\tilde{\Gamma}$ in $(C)_{n \times n}$ consists of scalar matrices. Then i) $\tilde{\Gamma} / \tilde{Z}$ is a finite group, ii) $\tilde{\Gamma}$ is an irreducible matric group and iii) $\tilde{\Gamma}$ is equivalent to a matric group whose elements are monomial matrices.

Proof. Denote by

$$
\tilde{Z}^{(r)}=\tilde{\Gamma} \supset \tilde{Z}^{(r-1)} \supset \cdots \supset \tilde{Z}^{(2)} \supset \tilde{Z}^{(1)} \supset \tilde{Z}^{(0)}=\{I\}
$$

the upper central series of $\tilde{\Gamma}$. We mean by $\chi(\tilde{\alpha}, \tilde{a})\left(\tilde{\alpha} \in \tilde{\Gamma}, \tilde{a} \in \tilde{Z}^{(2)}\right)$ the scalars such that

$$
(\tilde{\alpha}, \tilde{a})=\chi(\tilde{\alpha}, \tilde{a}) I . \quad\left(\tilde{\alpha} \in \tilde{\Gamma}, \tilde{a} \in \tilde{Z}^{(2)}\right) .
$$

Since

$$
\begin{aligned}
& (\tilde{\alpha} \tilde{\beta}, \tilde{a})=\tilde{\beta}^{-1}(\tilde{\alpha}, \tilde{a}) \tilde{\beta}(\tilde{\beta}, \tilde{a}) \\
& (\tilde{\alpha}, \tilde{a} \tilde{b})=(\tilde{\alpha}, \tilde{b}) \tilde{b}^{-1}(\tilde{\alpha}, \tilde{a}) \tilde{b}
\end{aligned}
$$

and

$$
\operatorname{det}(\tilde{\alpha}, \tilde{a})=1 \quad\left(\tilde{\alpha}, \tilde{\beta} \in \tilde{\Gamma} ; \tilde{a}, \tilde{b} \in \tilde{Z}^{(2)}\right),
$$

it follows that

$$
\begin{aligned}
\chi(\tilde{\alpha} \tilde{\beta}, \tilde{a}) & =\chi(\tilde{\alpha}, \tilde{a}) \chi(\tilde{\beta}, \tilde{a}), \\
\chi(\tilde{\alpha}, \tilde{a} \tilde{b}) & =\chi(\tilde{\alpha}, \tilde{a}) \chi(\tilde{\alpha}, \tilde{b}), \\
\chi\left(\tilde{\alpha}, \tilde{a^{n}}\right) & =\chi(\tilde{\alpha}, \tilde{a})^{n}=\operatorname{det}(\tilde{\alpha}, \tilde{a})=1 \\
& \left(\tilde{\alpha}, \tilde{\beta} \in \Gamma ; \tilde{a}, \tilde{b} \in \tilde{Z}^{(2)}\right) .
\end{aligned}
$$

This shows that $\tilde{\alpha} \tilde{a}^{n}=\tilde{a}^{n} \tilde{\alpha}\left(\tilde{\alpha} \in \Gamma, \tilde{a} \in \tilde{Z}^{(2)}\right)$, namely $\tilde{a}^{n} \in \tilde{Z}^{(1)}$ for $\tilde{a} \in \tilde{Z}^{(2)}$. Applying Lemma 1 to the quotient group $\tilde{\Gamma} / \tilde{Z}^{(1)}$. We conclude that the order of $\tilde{\Gamma} / \tilde{Z}^{(1)}$ is finite. Denote by $\Gamma$ the quotient group $\tilde{\Gamma} / \tilde{Z}^{(1)}$ and choose a system of representatives $\{\tilde{\alpha} \mid \alpha \in \Gamma\}$ in $\tilde{\Gamma}$, where $\tilde{\alpha}$ corresponds to $\tilde{\alpha}$. Then we get a 2-cocycle $\eta$ of $\Gamma$ with coefficients in the multiplicative group $C^{\times}$such that

$$
\tilde{\alpha} \tilde{\beta}=\eta(\alpha, \beta) \widetilde{\alpha \beta} \quad(\alpha, \beta \in \Gamma) .
$$


Since $\Gamma$ is a finite group, multiplying non-zero scalars $\lambda_{\alpha}$ to $\tilde{\alpha}$, we have a system of matrices $\left\{\mu_{\alpha}=\lambda_{\alpha} \tilde{\alpha} \mid \alpha \in \Gamma\right\}$ such that $\mu_{\alpha \beta} \mu_{\beta}^{-1} \mu_{\alpha}^{-1}(\alpha, \beta \in \Gamma)$ are roots of unity, Denote by $\Gamma^{*}$ the matric group generated by the matrices $\mu_{\alpha}(\alpha \in \Gamma)$. Then $\Gamma^{*}$ is a finite group of matrices such that the commutor of $\Gamma^{*}$ in $(C)_{n \times n}$ consists of scalar matrices. This means that $\Gamma^{*}$ is an irreducible matric group. Since $\Gamma^{*}$ is a finite nilpotent group, the irreducibility of $\Gamma^{*}$ implies that $\Gamma^{*}$ is equivalent to a matric group whose elements are monomial matrices ${ }^{1}$.

3. We now prove Theorem 1 . Let $\mathscr{D}$ be a complex analytic manifold and let $\Gamma$ be a finitely generated nilpotent subgroup in the group of automorphisms of $\mathscr{D}$ such that $\Gamma$ acts properly discontinuously on $\mathscr{D}$ without fixed points. Let $\varphi$ be the natural map $\mathscr{D} \rightarrow \mathscr{D} / \Gamma$ and let $E$ be a holomorphic vector bundle over $\mathscr{D} / \Gamma$ such that i) the inverse image $\varphi^{*}(e)$ of $E$ is trivial, ii) the associated projective bundle $P(E)$ is locally flat, and iii) $E$ is simple. The inverse image $\varphi^{*}(E)$ can be identified with $\mathscr{D} \times C^{n}$ and the automorphisms $\alpha \in \Gamma$ of $\mathscr{D}$ induce bundle automorphisms

$$
(z, v) \rightarrow\left(z \alpha, v \mu_{\alpha}(z)\right) \quad(\alpha \in \Gamma),
$$

where $\mu_{\alpha}(z)(\alpha \in \Gamma)$ are holomorphic $n \times n$-matric functions such that

1) $\operatorname{det} \mu_{\alpha}(z) \neq 0$ everywhere on $\mathscr{D}$,

2) $\mu_{\alpha}(z) \mu_{\beta}(z \alpha)=\mu_{\alpha \beta}(z),(\alpha, \beta \in \Gamma)$

The local flatness of $P(E)$ is equivalent to

3) $\mu_{\alpha}(z)=\mu_{\alpha} \xi_{\alpha}(z) \quad(\alpha \in \Gamma)$ with scalar functions $\xi_{\alpha}(z)$ and constant $n \times n$-matrices $\mu_{\alpha}$.

The simplicity of $E$ is equivalent to

4) the commutor of $\left\{\mu_{\alpha} \mid \alpha \in \Gamma\right\}$ in $(C)_{n \times n}$ consists of scalar matrices.

Let $\tilde{\Gamma}$ be the matric group generated by $\left\{\mu_{\alpha} \mid \alpha \in \Gamma\right\}$ and let $\tilde{Z}$ be its center. Then from 2) and 3) the quotient group $\tilde{\Gamma} / \tilde{Z}$ is isomorphic to a quotient group of $\Gamma$, and thus $\tilde{\Gamma} / \tilde{Z}$ is finitely generated. Therefore by virtue of Lemma $2, \tilde{\Gamma}$ is a matric group such that i) $\tilde{\Gamma} / \tilde{Z}$ is a finite group, ii) $\tilde{\Gamma}$ is an irreducible matric group and iii) $\tilde{\Gamma}$ is equivalent to a group of monomial matrices. After suitable change of the base of the vector space $C^{n}$, we may assume that $\mu_{\alpha}(\alpha \in \Gamma)$ are monomial matrices. Denote by $\mu_{\alpha}^{*}$ the $n \times n$-matrix obtained by replacement of non-zero entries of $\mu_{\alpha}$ with 1 . Then $\Gamma^{*}=\left\{\mu_{\alpha}^{*} \mid \alpha \in \Gamma\right\}$ form a group of

1) See [1] VII 52. 1. 
permutation matrices. Since the matric group $\tilde{\Gamma}$ is irreducible the permutation group $\Gamma^{*}$ is transitive. If we denote by $\Delta$ the subgroup of $\Gamma$ consisting of $\alpha$ such that

$$
\mu_{\alpha}^{*}=\left(\begin{array}{ll}
1 & 0 \\
0 & *
\end{array}\right),
$$

then from the transitivity we can conclude $[\Gamma: \Delta]=n$. If we decompose $\mu_{r}(z)$ as

$$
\mu_{\gamma}(z)=\left(\begin{array}{cc}
\nu_{r}(z) & 0 \\
0 & \mu_{\gamma}^{(1)}(z)
\end{array}\right) \quad(\gamma \in \Delta),
$$

then the group $\Delta$ acts on $\mathscr{D} \times C$ and $\mathscr{D} \times C^{n-1}$ as follows

$$
(z, u) \rightarrow\left(z \gamma, u \nu_{r}(z)\right)
$$

and

$$
(z, v) \rightarrow\left(z \gamma, v \mu_{r}^{(1)}(z)\right) \quad(\gamma \in \Delta) .
$$

Using these actions of $\Delta$ we get a line bundle $L$ and a vector bundle $E^{(1)}$ of rank $n-1$ over $\mathscr{D} / \Delta$ as the quotients

$$
L=\mathscr{D} \times C / \Delta
$$

and

$$
E^{(1)}=\mathscr{D} \times C^{n-1} / \Delta
$$

such that

$$
\psi^{*}(E)=L \oplus E^{(1)},
$$

where $\psi$ is the natural map $\mathscr{D} / \Delta \rightarrow \mathscr{D} / \Gamma$. Taking the direct images of of both sides, we have

$$
E \overbrace{\oplus \cdots \oplus}^{n} E=\psi_{*} \psi^{*}(E)=\psi_{*}(L) \oplus \psi_{*}\left(E^{(1)}\right) .
$$

Since $[\Gamma: \Delta]=n$ and the linear hull of $\left\{\mu_{\alpha} \mid \alpha \in \Gamma\right\}$ is the full matric ring $(C)_{n \times n}, \psi_{*}(L)$ is simple and $\psi_{*}(L)=n$. By the Krull-Remark-Schmidt theorem for vector bundles,

$$
E \simeq \psi_{*}(L) .
$$




\section{REFERENCES}

[1] Curtis and Reiner, Representation theory of finite groups and associative algebras, New York/London, 1962.

[2] H. Morikawa, A note on holomorphic vector bundles over complex tori, Nagoya Math. J. Vol. 41 (1170), 101-106.

Nagoya University 